\title{
Research on Semiology of Role Model in China's Paper-Cutting Animation
}

\author{
Yin Meiying \\ Shanghai University of Engineering Science, China \\ seven840523@163.com
}

Keywords: Paper-cut animation, Semiotics, Animation modeling.

\begin{abstract}
In this paper, China's paper-cutting animation theories are teased out by making use of the study methods related to semiology, by which a new level for studying China's paper-cutting animation theories was achieved. From the view of symbolic features and cultural connotation, this paper studies China's paper-cutting animation, by which the deep structure of China's paper-cutting animation and meanings of visual language symbols of China's paper-cutting animation can be looked into. At the same time, the study can also provide guidance for the practices linking with China's paper-cutting animation. By introducing research methods of semiology aesthetics, the development direction of China's paper-cutting animation can be understood by the symbols of China's paper-cutting animation. Then discuss the relationship between China's paper-cutting and paper-cutting animation, the meaning of paper-cutting symbols in animation.
\end{abstract}

\section{Current situation of China's paper-cutting animation}

China's paper-cutting animation once played an important role in the world. In 1958, Wan Guchan, an art film artist, together with some other young art film workers, made the first paper-cutting animation in China --- Zhu Bajie Eats Watermelon. Later, under his leadership, some other paper-cutting animations were completed, including $Y u$ Tong, Jigong Fights Crickets, Ginseng Baby and Golden Conch etc, by which he was rewarded with many domestic and foreign awards. Actually, paper-cutting animation developed a new field for the art films. However, in 1990s, China's animation, including paper-cutting animation, suffered a severe downturn. Luckily, today, the government provides great supports for developing paper-cutting animation and the investment is being increased. That is China's paper-cutting animation is witnessing unprecedented opportunities for its further development.

\section{Overview of China's paper-cutting animation}

Paper-cutting animation, also named as paper-cutting films, is a kind of art film learning from some traditional arts like Chinese shadow play and folk paper-cutting. Normally, the background of paper-cutting consists of the paper, the front views and rare views posted on the glass. The glass plates shall be located with a certain distance between each two ones so that the light arrangement can be done hierarchically. Then, the paper toy can be horizontally lie down on the glass plates and stop-motion shooting can be done. This is a traditional way to make paper-cutting animation. These days, digital media technology is being used to help with the paper-cutting modelling, which will greatly enhance the quantity and visual quality of paper-cutting animation.

Animation is a product whose ideas can be transferred to economic benefits. Normally, characters existed in the animations can give out physical products continuously, which is closely linked with the characters modeling in the animation. In fact, animation image is a kind of symbolic transmission. That is when people enjoy an animation, they will love the characters in the animation. In this way, symbolic elements can realize their own industrial value. Though animation images are fictitious and nonobjective, which are always being exaggerated, the images are provided with humanistic feelings. Therefore, they not only represent their own values but also touch the deep inner heart of humans. Animation image is a kind of symbol, which can also be considered to be a symbol set including the common symbols shared by cultural symbols and commercial symbols. 
Since animation image can play a significant part in propagandizing paper-cutting culture and paper-cutting in China, how to develop the animation image symbols existed in China's paper-cutting animation can provide us with enlightenment.

\section{Semiology and paper-cutting animation}

From the view of semiology, this paper studies the relationship between China's paper-cutting animation and modeling and the techniques of expression by discussing China's paper-cutting animation and performing comparative analysis. Major symbols, the modeling and stories of China's paper-cutting animation, show us the historical changes of story symbols and modeling symbols existed in China's paper-cutting animation, as well as the sources of materials. In the aspect of plot symbols and theme symbols, the story features of animation at each stage are discussed; from the aspect of graphic symbols, narrative symbols and symbolic signs, the modeling of animation are analyzed.

\section{Classification of paper-cutting animation semiology}

For a long time, studies about Chinese animation theory have been restricted by the techniques. Especially that a series of studies in the field of China's paper-cutting are not accessed to, including culture of China's paper-cutting animation, paper-cutting animation images and scripts of paper-cutting animation stories. Instead, the studies about China's paper-cutting animation were all done from the view of semiology. Then visual language symbols existed in China's paper-cutting animation were teased out. By extracting and analyzing the symbols, we can deeply explore the cultural connotation represented by the images of China's paper-cutting animation. This is what we need to think about first that whether we should use semiology theoretical research methods when studying China's paper-cutting animation.

Actually, studying the modeling of paper-cutting animation from the view of semiology is quite interesting. Normally, the implied meanings represented by the paper-cutting will be shown by the paper-cutting animation, whose direct or indirect meanings of the symbols will be delivered by paper-cutting animation.

A Mouse Marriage is a widely-known folk story, which has become a popular material used by the folk art. It is known that rats are quite reproductive and rats are honored as "God of Offspring", this is why they rank the first place among all the twelve Chinese zodiac signs. In China, Jan. 7 (lunar calendar) is named as "Human Day" and there is a folk customs that rats' daughters are to get married on that day. In fact, the marriage of the rats has the meaning of procreation. A Mouse Marriage, the paper-cutting animation, shows the audiences that if one tells lies and performs corruptions, he or she will be punished and put into the prison. Anyway, this paper-cutting animation discloses the greediness and cattiness of the humans.

Table 1 Symbolic Meanings of Role Models in A Mouse Marriage

\begin{tabular}{|c|c|c|c|c|}
\hline Roles & Little Gray Rat & After Dress-up & Rat Mother & Rat Father \\
\hline Signifier & & Cocky, \\
\hline Signified & Poor, honest, naive & $\begin{array}{c}\text { Cotenty } \\
\text { pretentious }\end{array}$ & Cunning and catty \\
\hline
\end{tabular}

In A Mouse Marriage, the Little Gray Rat is poor young man who is not self-confident. After dress-up, he becomes a Little White Rat, from whom we can feel his cockiness and pretentiousness. For the Rat Mother, he wears a clothes with the sign of money, from which we can know that she is rich or she is greedy. Rat Father wears a pair of small glasses. Normally, we know that in other 
cartoons, the man wearing a pair of glasses often knowledgeable and civilized. However, in A Mouse Marriage, the Rat Father is quite cunning and catty. By using her daughter, Rat Father requires the Little Gray Rat to get the cake, which means "corruption".

Table 2 Molding in A Mouse Marriage

\begin{tabular}{|c|c|c|}
\hline Scene & Marriage \\
\hline Signifier & In the Cage \\
\hline Signified & The peach blossom is a symbol of love and \\
marriage
\end{tabular}

In A Mouse Marriage, peach blossom is a symbol of love. Little Gray Rat is going to get married to Miss. Rat and the wedding procession is playing instruments to celebrate the special happy moment. However, Rat Mother gets greedy again when she saw that there are cakes in another wedding procession. So she requires the Little Gray Rat to prepare cakes if he wants to marry her daughter. In order to steal the cakes, Little Gray Rat is going to face a fate that he is to be trapped in the rat-catching cage, which means being put into prison.

When modeling the terrine, the traditional rules are broken --- visual perspective is not used any more. The entrance of the terrine is round while the bottom is flat and straight. Such a modeling is well explained by the rules used by folk paper-cutting. Only a terrine whose bottom is flat and straight can be put stably. A terrine with round entrance can access to the sky. Actually, such a terrine is consistent with the philosophy of "round heaven and square earth", which also stands for the mothers. The classical models of folk paper-cutting are directly used in the animations. The landscapes indoor and outdoor will not mutually affected. In this way, the whole space and environment will be presented to the audiences. Although this does not comply with perspective principles, it has been a fixed technique of expression by paper-cutting art. By now, this has been widely accepted by the public. Imagery modeling is a kind of modeling method used by folk paper-cutting, which is that the folk artist uses some events and stuffs which seem to have no link with each other to present new conceptions and meanings.

Table 3 Symbolic Meanings of Major Models in Fishing Child

\begin{tabular}{|c|c|c|c|c|}
\hline Models & Fishing Child & Fish & Lotus Blossom & White marble fish terrine \\
\hline Signifier & &
\end{tabular}

For folk art, fish and lotus are the frequently used materials because they are the symbol of life (fish and lotus are quite reproductive). For folk paper-cutting, dermatoglyphic patterns like Fish Play with Lotus and Lotus Giving Birth to Babies etc are used to express our best wishes for life. In Fishing Child, $\mathrm{Yu}$ Tong was born in the lotuses, who is the embodiment of innocence, holiness, bravery and wisdom. Actually, this modeling view is originated from the imagery modeling of folk paper-cutting. In Fishing Child, The golden fish is in the form of paper-cutting, figures and some scenes are in the style of New Year's pictures (green willow New Year's pictures). When Yu Tong holds his fishing rod to spray the beads, golden fish is the focus. The white marble fish terrine is obtained by the fishing men in China's sea area. This can be known from his argument with the foreign priest that "you said that this was made by your country, but the fishing kid on it is a 
Chinese and what he wears is quite Chinese style. Why?" From what the fishing man says, we can vividly know about the modeling of the fish terrine. Especially that Yu Tong is shaped as a classical kid of old times in China --- big eyes and bushy eyebrows, lotus-root-like arms and legs, black hair with fringe (one of the two twisted knots of hair on each side of the head), and Yu Tong wears a red bellyband. In a word, Yu Tong is a popular Chinese baby. The action breaking the fish terrine implies that China is fallen apart and the masses has no means to live. When the fish terrine is being broken, there comes a miracle --- Yu Tong comes out from the terrine and uses his fishing rod to do havoc. And $\mathrm{Yu}$ Tong throws the foreign priest into the sea.

From the models in Fishing Child, we can know that the design of animation modeling shall have proper and unique modeling tone and modeling language, trying to make modeling design become the organic element of animation. In Fishing Child, the modeling language of modeling language provides the audiences a unique visual image, which shows the techniques and skills when making Yu Tong.

\section{Conclusion}

Based on the theoretical framework of interaction between signifier and signified, China's paper-cutting animations are divided in terms of their symbolic signifier and signified, by which a new mode for acknowledging the role symbols existed in China's paper-cutting animation. The color is from the reflection of lights, which is produced by the co-action among propor color, illuminant color and ambient color. However, this is abandoned by the folk art. Instead, the paper-cutting artists will present the implied meanings (blessing, benefit-seeking and evil-avoiding) represented by the colors via their own understanding and feelings to the colors. And as time goes by, such implied meanings are kept and inherited. Under the condition not disobeying the symbolic meanings of color culture, China's paper-cutting attaches great emphasis to the visual effect of colors. Colors are used according to the association of colors and emotions. Actually, the attitude to the color is also used by China's paper-cutting animation.

China's paper-cutting symbols can directly show us the implied meanings existed in folk paper-cutting. Therefore, it is quite important to a role which is consistent with the plots of the animation. Although it is not difficult to find out the proper role from China's paper-cutting symbol bank, it is significant to have a deep understanding on the implied meaning of the paper-cutting symbols. It is key to apply the China's paper-cutting models to the paper-cutting animations.

\section{Acknowledgment}

This paper is one of the periodical achievements made by the project Research on Semiology of China's Paper-cutting Animation (School Start-up 2016-24) carried out by Shanghai University of Engineering Science.

\section{References}

[1] Li Yongjie, Zhang Bingyue, Wang Zhiting, Jiang Jiping. Design and Implementation of Paper-cut Animation Based on Digital Media Technology - A Case Study of Original Works "Spring Festival Custom" [J]. Information and Computer (Theoretical Edition), 2017, (08): 167-169.

[2] Yang Yang. Paper-cut animation and cultural heritage of the new media era [J]. Today media, 2017,25 (02): 151-152.

[3] Qin slowly. Chinese paper cutting in the use of animation art creation [J]. Western Leather, 2016,38 (06): 40. 